\title{
Captures
}

Figures, théories et pratiques de l'imaginaire

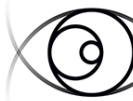

C A P T U R E S

\section{Volume 2 Numéro 2}

Une revue de recherche

\section{Vincent Lavoie}

Volume 2, Number 2, 2017

Imaginaire de la ligne

URI: https://id.erudit.org/iderudit/1059765ar

DOI: https://doi.org/10.7202/1059765ar

See table of contents

Publisher(s)

Figura, Centre de recherche sur le texte et l'imaginaire

ISSN

2371-1930 (digital)

Explore this journal

Cite this document

Lavoie, V. (2017). Volume 2 Numéro 2 : une revue de recherche. Captures, 2(2). https://doi.org/10.7202/1059765ar

\section{Article abstract}

Avec la parution de ce quatrième numéro, Captures atteint sa pleine maturité au titre de revue de recherche. Plus de 36 contributions savantes publiées à ce jour, présentant les plus récents travaux de chercheuses et de chercheurs du Québec, du Canada, des États-Unis, de la France, de la Belgique, de l'Angleterre et de la Norvège, en témoignent avec éloquence. Le formidable accueil que reçoit Captures depuis sa création est attribuable à plusieurs facteurs...
Tous droits réservés @ Vincent Lavoie, 2017

\section{(c) (i) $\Theta$}

This document is protected by copyright law. Use of the services of Erudit (including reproduction) is subject to its terms and conditions, which can be viewed online.

https://apropos.erudit.org/en/users/policy-on-use/ 
Version enrichie de cet article : http://revuecaptures.org/node/1017

\section{Volume 2 Numéro 2}

\section{Une revue de recherche}

\section{Vincent Lavoie}

Résumé :

Avec la parution de ce quatrième numéro, Captures atteint sa pleine maturité au titre de revue de recherche. Plus de 36 contributions savantes publiées à ce jour, présentant les plus récents travaux de chercheuses et de chercheurs du Québec, du Canada, des États-Unis, de la France, de la Belgique, de l'Angleterre et de la Norvège, en témoignent avec éloquence. Le formidable accueil que reçoit Captures depuis sa création est attribuable à plusieurs facteurs...

With the launch of this fourth issue Captures reaches its full maturity as a research journal. More than 36 scientific contributions have been published, and counting. They present the most recent research results by specialists from Quebec, Canada, the United States, France, Belgium, England. The exceptional interest with which the academics have welcomed Captures into their world can be related to multiple factors...

Avec la parution de ce quatrième numéro, Captures atteint sa pleine maturité au titre de revue de recherche. Le nombre de contributions savantes publiées à ce jour — plus de 36 présentant les plus récents travaux de chercheuses et de chercheurs du Québec, du Canada, des États-Unis, de la France, de la Belgique, de l'Angleterre et de la Norvège - témoigne avec éloquence de la solidité des assisses de notre revue. Le formidable accueil que reçoit Captures depuis sa création est attribuable à plusieurs facteurs. La crédibilité du regroupement stratégique qui l'abrite, Figura, le Centre de recherche sur le texte et l'imaginaire, en est assurément un de taille. Le processus indépendant d'évaluation à l'aveugle des articles soumis en est un autre de la plus grande importance, puisqu'il est garant de la légitimité scientifique de la revue. Assuré par des experts d'ici et d'ailleurs, celui-ci est conduit dans le respect des critères de validation de la recherche scientifique que sont l'originalité, la pertinence, la qualité et le caractère inédit des travaux. Ces critères illustrent parfaitement le mandat que Captures s'est donné.

Le présent numéro, entièrement consacré à l'« Imaginaire de la ligne », thème du dossier dirigé par Véronique Cnockaert, poursuit cette lancée. Prenant acte des travaux fondateurs de l'anthropologue anglais Tim Ingold (Une brève histoire des lignes, 2011), les articles réunis dans ce dossier mettent au jour la puissance ordonnatrice de la ligne. Ligne ascendante ou descendante d'une destinée, lignes du terrain de jeu, ligne morale ou idéologique, ligne de vie ou de tir souvent déviée, lignes de force ou de faille, méridienne ou du temps, ainsi que l'énonce Véronique Cnockaert, l'on comprend, à la lecture de ces contributions, combien ces 
tracés, métaphoriques ou réels, sont omniprésents. La littérature, bien entendu, est en la matière riche d'occurrences. II en va de même de l'architecture, ainsi qu'en atteste l'entretien inédit accordé à Captures par Rudy Ricciotti, architecte, entre autres, du MuCEM (Musée des civilisations à Marseille) et inventeur de la ligne dite en « os de poulet ». Les arts visuels ne sont pas en reste, exploitant ou critiquant eux-aussi les pouvoirs de la ligne. Le montrent à l'évidence les propositions artistiques figurant dans la section «Contrepoints », l'une des tribunes de la revue dédiés à la création visuelle et littéraire. Tout comme les œuvres de Bertrand R. Pitt, à qui l'on doit la signature visuelle de ce numéro.

Vincent Lavoie

Directeur 